\title{
ACKNOWLEDGMENTS
}

\section{Reviewers for Journal of Hematopathology in 2021}

Published online: 1 March 2022

(c) Springer-Verlag GmbH Germany, part of Springer Nature 2022

The following people donated their time and expertise as peer reviewers of manuscripts submitted to the Journal of Hematopathology in 2021. The journal thanks them all for their invaluable contributions. An asterisk follows the name of those reviewers who evaluated three or more manuscripts in 2021.
Patrick Adam, MD

Archana M Agarwal, MD

Nidhi Aggarwal, MD

Catalina Amador, MD

Katsuyuki Aozasa, MD

Mary Ann Thompson Arildsen, MD, PhD

Aditya Badheka, MBBS, MS

Nathanael G. Bailey, MD

Mohammad I. Barouqa, MD

Carlos Barrionuevo, MD, PhD

Siddharth Bhattacharyya, MD

Shweta Bhavsar, MD

Darci Block, PhD

Daniel Boyer*, MD, PhD

Noah Brown, MD

José Cabeçadas, MD

Gabriel C. Caponetti, MD

Eric D. Carlsen, MD, PhD

Luis Carrillo, MD

Alexander C.L. Chan, MD

Shweta Chaudhary, MD

Ding-Bao Chen, MD

Dong Chen, MD, PhD

Weina Chen, MD, PhD

Irina Chibisov, MD

Elizabeth Courville, MD

Magdalena B. Czader, MD, PhD

Daphne de Jong, MD, PhD

Laurence de Leval, MD, PhD

Amy Susan Duffield, MD, PhD

Ashley M. Eckel, MD, PhD

Mohsen Saleh Elalfy, MD, PhD

Mark D. Ewalt, MD

Andrew L. Feldman, MD

Falko Fend, MD

Judith A. Ferry, MD

\author{
Ali Gabali, MD, PhD \\ David Gailani, MD \\ Juan Gomez-Gelvez, MD \\ Julia T. Geyer, MD \\ Béatrice Gulbis, MD, PhD \\ Jonathon H. Gralewski, DO \\ Patricia T. Greipp, DO \\ Bartosz Grzywacz, MD \\ Timothy M. Hanley, MD, PhD \\ Marian H. Harris, MD, PhD \\ Robert P. Hasserjian, MD \\ Nahla M. Heikal, MD \\ John M. Higgins, MD \\ Bo Hong, MD \\ Sarika Prakash Jain, MD \\ Ridas Juskevicius, MD \\ Kristin H. Karner, MD \\ David F. Keren, MD \\ Rebecca L. King, MD \\ Monika Klimkowska*, MD, PhD \\ Alexandra Kovach*, MD \\ Shaji Kumar, MD \\ Daniel Larson, MD \\ Terra L. Lasho, PhD \\ Megan Lim, MD, PhD \\ Yen-Chun Liu, MD, PhD \\ Abner Louissaint, MD, PhD \\ William R. Macon, MD \\ Emily F. Mason, MD, PhD \\ Maurizio Martini, MD, PhD \\ Rodney Miles, MD, PhD \\ Roberto N. Miranda, MD \\ Kamran Mirza, MD, PhD \\ Erika Moore, MD \\ Karen A. Moser, MD \\ Megan O. Nakashima, MD
}

Amrom Obstfeld, MD, PhD

Sarah L. Ondrejka, DO

Oyesola Oyewole Ojewunmi, PhD

Horatiu Olteanu, MD, PhD

Nisha Patel, DO

Jennifer Picarsic, MD

Julianne Qualtieri, MD

Leticia Quintanilla-Fend, MD, PhD

Phil Raess, MD, PhD

Aishwarya Ravindran, MBBS

Bryan Rea, MD

Eduardo Rego, MD, PhD

Kaaren Reichard, MD

Lisa Rimsza, MD

Flavia G. Rosado, MD

Daniel E. Sabath, MD, PhD

Sam Sadigh, MD

Adam Seegmiller, MD, PhD

Jansen N. Seheult, MB BCh BAO, MD

Min Shi, MD, PhD

Craig R. Soderquist, MD

Aliyah R. Sohani, MD

Zhenya Tang, MD, PhD

Karl Theil, MD

Reha Toydemir, MD, PhD

José Vassallo*, MD, PhD

Gerald B. Wertheim, MD, PhD

Adam Wilberger, MD

Xinjie $\mathrm{Xu}, \mathrm{PhD}$

Mariko Yabe, MD, PhD

Ji Yuan, MD, PhD

Maria Claudia Nogueira Zerbini, MD, PhD

Publisher's note Springer Nature remains neutral with regard to jurisdictional claims in published maps and institutional affiliations. 